\title{
ON THE NONLINEAR IMPLICIT COMPLEMENTARITY PROBLEM
}

\author{
A.H. SIDDIQI and Q.H. ANSARI \\ DEPARTMENT OF MATHEMATICS \\ ALIGARH MUSLIM UNIVERSITY \\ ALIGARH-202002 \\ INDIA
}

(Recelved March 19, 1992 and in revised form July 10, 1992)

ABSTRACT. In this paper, we consider a new class of implicit complementarity problem and study the existence of its solution. An iterative algorithm is also given to find the approximate solution of the new problem and prove that this approximate solution converges to the exact solution. Several special cases are also discussed.

KEY WORDS AND PHRASES. Complementarity problem, Fixed point. AMS (1980), SUBJECT CLASSIFICATION CODES. 49A29, 90C33, 65K10, 47H10., $49 \mathrm{~A} 10$.

\section{INTRODUCTION.}

Due to the applications in areas such as, Optimization Theory, Engineering, Structural Mechanics, Elasticity, Lubrication Theory, Economics, Variational Calculus, Equilibrium Theory on Networks, Stochastic Optimal Control etc., the complementarity problem is one of the interesting and important problems which was introduced by G.E. Lemke in 1964, but Cottle [1] and Cottle and Dantzig [2] formally defined the linear complementarity problem and called it the fundamental problem. In recent years, it has been generalized and extended to many different directions. The implicit complementarity problem which is one of the generalizations of complementarity problem, was imposed by some special problems in Stochastic Optimal Control and it was considered by Bensoussan, Goursat and Lions [3], Bensoussan and Lions [4, 5, 6], Dolcetta and Mosco [7], Isac [8, 9, 10] and Mosco [11]. In 1987, M.A. Noor [12] considered and studied an important and useful generalization of complementarity problem which is called mildly nonlinear complementarity problem.

The recent research carried out in this field motivated us to introduce and study a new class of complementarity problem which includes implicit complementarity problem and mildly nonlinear complementarity problem as special cases. By using the technique of Isac [9] we prove an existence theorem. An algorithm is also given to find the approximate solution of the new complementarity problem and prove that this approximate solution converges to the exact solution.

2. MORLULATION AND BASIC RESULTS.

Let $\mathrm{H}$ be $\mathrm{a}$ Hilbert space with norm and inner product denoted by $\|\cdot\|$ and <...>, respectively and $\mathrm{K}$ be a closed convex Cone of $\mathrm{H}$. If $T$ and $A$ are nonlinear operators from $\mathrm{H}$ into itself then the mildly nonlinear complementarity problem (M.N.C.P.) which is introduced and studied by Noor [12], is to find $u \in K$ such that

$$
T(\dot{x})+A(x) \varepsilon K^{*} \text { and } \alpha, T(x)+A(x)>=0 \text {, }
$$


where we denote $K^{*}$ the polar cone of $K$, that is, $\mathrm{K}^{\star}=\{\mathrm{x} \in \mathrm{K} /\langle\mathrm{x}, \mathrm{y}\rangle \geq 0$ for all $\mathrm{y} \in \mathrm{K}\}$.

If $\mathrm{DCH}$ is a subset of $\mathrm{H}$ and $\mathrm{G}: \mathrm{D} \rightarrow \mathrm{H}$ then the implicit complementarity problem (I.C.P.) is to find $x \in D$ such that

$$
\mid \begin{aligned}
& G(x) \in K, T(x) \in K^{\star} \text { and } \\
& \measuredangle G(x), T(x)>=0
\end{aligned}
$$

Now we consider a more general form of complementarity problem for which (M.N.C.P) and (I.C.P.) are special cases.

$$
\begin{array}{l|l}
\text { (S.N.I.C.P.) } & \begin{array}{l}
\text { Find } x \in D \text { such that } \\
G(x) \in K, T(x)+A(x) \in K^{*} \text { and } \\
G(x), T(x)+A(x)>=0
\end{array}
\end{array}
$$

We shall call it strongly nonlinear implicit complementarity problem (S.N.I.C.P.).

We recall that if $P_{K}$ denotes the projection of $H$ onto $K$, that is, for every $x \in H, P_{K}(x)$ is the unique element satisfying:

$$
\left\|x-P_{K}(x)\right\|=\underset{y \in K}{\min }\|x-y\|
$$

then we have the following result.

PROPOSITION 2.1 [13]. For every element $x \in H, P_{K}(x)$ is characterised by the following properties:

(i) $<P_{K}(x)-x, y>0$, for all $y \in K$,

(ii) $<P_{K}(x)-x, P_{K}(x)>=0$.

DEFINITION 2.1 [9]: Given a subset $\mathrm{D} \subset \mathrm{H}$, we consider the mappings $\mathrm{F}, \mathrm{G}: \mathrm{D} \rightarrow \mathrm{H}$; $\phi, \Psi: R_{+} \rightarrow R_{+}$and we say that:

(a) F is a $\phi$-Lipschitz mapping with respect to $G$ if

for all $x, y \in D$,

$$
\|F(x)-F(y)\| \leq\|G(x)-G(y)\| \phi(\|G(x)-G(y)\|):
$$

(b) $\mathrm{F}$ is a $\Psi$-strongly monotone mapping with respect to $G$ if

for all $x, y \in D$.

$$
\langle F(x)-F(y), G(x)-G(y)\rangle \geq|| G(x)-G(y) \|^{2} \Psi(|| G(x)-G(y) \|) \text { : }
$$

If in Definition 2.1, $G(x)=x$, for all $x \in D$ then we say that $F$ is a $\phi$-Lipschitz mapping (respectively, $F$ is a $\Psi$-strongly monotone mapping).

Obviously, if $\phi$ and $\Psi$ are strictly positive constants, we obtain from Definition $2.1(a)$ (respectively (b)) that $F$ is a Lipschitz continuous (respectively, strongly monotone) mapping.

DEFINITION 2.2 [14]: A metric space $(X, \rho)$ is said to be metrically convex, if for each $x, y \in x,(x \neq y)$ there is a $z \neq x, y$ for which $\rho(x, y)=\rho(x, z)+\rho(z, y)$.

We denote, $P=\{\rho(x, y) / x, y \in x\}$.

TFEOREM $2.1[14]$ : Let $(x, \rho)$ be a complete metrically convex metric space. If for the mapping $F: X \rightarrow X$ there is a mapping $\phi: P \rightarrow R_{+}$satisfying, 
1. $\rho(F(x), F(y)) \leq \phi(\rho(x, y))$, for all $x, y \in x$,

2. $\phi(t)<t$, for all $t \in \overline{\mathrm{P}} \backslash\{0\}$.

then $F$ has a unique fixed point $x_{0}$ and $T^{n}(x) \rightarrow x_{0}$ for each $x \in X$.

3. EXISTENCE THEORY

THEOREM 3.1. Assume that

(i) $T$ is $\phi$-Lipschitz mapping with respect to $G$;

(ii) $\mathrm{T}$ is $\Psi$-strongly monotone mapping with respect to $\mathrm{G}$;

(iii) A is V-Lipschitz mapping with respect to $G$;

(iv) $\mathrm{K} \subset \mathrm{G}(\mathrm{D})$;

(v) there exists a real number $\xi>0$ such that,

$$
\xi \phi^{2}(t)-\frac{\nu^{2}(t)}{\xi}+\frac{2 \nu(t)}{\xi}<2 \Psi(t)<\frac{1}{\xi}+\xi \phi^{2}(t),
$$

for all $t \in R_{+}$.

Then the strongly nonlinear implicit complementarity problem has a solution.

Moreover, if $\mathrm{G}$ is one-to-one, then the problem (S.N.I.C.P.) has a unique solution.

PROOF. We consider the mappings $p$ and $q: K \rightarrow H$ (which are not unique) defined by

$$
\mid \begin{aligned}
& \mathrm{p}(\mathrm{u})=\mathrm{T}(\mathrm{x}) \text { and } \mathrm{q}(\mathrm{u})=\mathrm{A}(\mathrm{x}) \\
& \text { where } \mathrm{x} \in \mathrm{G}^{-1}(\mathrm{u})=\{\mathrm{x} \in \mathrm{D} / \mathrm{G}(\mathrm{x})=\mathrm{u}\} \\
& \text { and } \mathrm{u} \in \mathrm{K} .
\end{aligned}
$$

From this definition, we observe that $p$ and $q$ have the following properties:

(vi) $\quad|| p(u)-p(v) \| \leq|| u-v|| \phi(|| u-v \mid \|)$.

for all $u, v \in K$

(vii) $\langle\mathrm{p}(\mathrm{u})-\mathrm{p}(\mathrm{v}), \mathrm{u}-\mathrm{v}\rangle \geq|| \mathrm{u}-\mathrm{v}||^{2} \Psi(|| \mathrm{u}-\mathrm{v}||)$,

for all $u, v \in k$

(viii) ||$q(u)-q(v) \| \leq|| u-v|| v(|| u-v||)$

for all $u, v \in K, v: R_{+} \rightarrow R_{+}$.

Now, we conclude that the S.N.I.C.P. is equivalent to the M.N.C.P.

$$
\begin{array}{l|l}
\text { (M.N.C.P.) } & \begin{array}{l}
\text { Find } u \in K \text { such that } \\
p(u)+q(u) \varepsilon K^{*} \text { and }\langle u, p(u)+q(u)\rangle=0
\end{array}
\end{array}
$$

By Proposition 2.1, it is easy to prove that the S.N.C.P. has a solution if and only if the mapping $\mathrm{F}: \mathrm{K} \rightarrow \mathrm{K}$ defined by

for all $u \in K$,

$$
F(u)=P_{K}(u-\xi(p(u)+q(u)),
$$

has a fixed point (where $\xi$ is the real number used in assumption (V)).

We, now will show that $T$ has a fixed point.

$$
\begin{aligned}
\|F(u)-F(v)\| & =\left\|p_{K}(u-\xi(p(u)+q(u)))-p_{K}(v-\xi(p(v)+q(v)))\right\| \\
& \leq|| u-\xi(p(u)+q(u))-v+\xi(p(v)+q(v)) \| \\
& \leq|| u-v-\xi(p(u)-p(v))||+\xi|| q(u)-q(v)||
\end{aligned}
$$


since $P_{K}$ is nonexpansive [15].

By (vi), (vii) and (viii), we obtain

$$
|| u-v-\xi(p(u)-p(v)) \| \leq|| u-v||\left[1-2 \xi \Psi(|| u-v||)+\xi^{2} \phi^{2}(|| u-v||)\right]^{\frac{1}{2}}
$$

and

$$
|| q(u)-q(v)|| \leq|| u-v|| v(|| u-v||)
$$

Therefore,

$$
|| F(u)-F(v)|| \leq|| u-v||\left[\left\{1-2 \xi \Psi(|| u-v||)+\xi^{2} \phi^{2}(|| u-v||)\right\}^{1}+v(|| u-v||)\right]
$$

If we denote

$$
\phi(t)=t\left[\left\{1-2 \xi \Psi(t)+\xi^{2} \phi^{2}(t)\right\}^{\frac{1}{2}}+v(t)\right],
$$

for all $t \in R_{+}$"

we observe, by assumption ( $v)$ and the fact that a Hilbert space is a complete metrically convex metric space, that all assumptions of Theorem 2.1 are satisfied. $\left(\bar{p}=R_{+}\right)$. Hence $F$ has a unique fixed point $u_{0}$ and $F^{n}(u) \rightarrow u_{0^{\prime}}$ for every $u \varepsilon K$.

Obviously, if $G$ is one-to-one mapping then S.N.I.C.P. has a unique solution.

COROLIARY 3.1. Assume that

(i) $T$ is $\phi$-Lipschitz and $\Psi$-strongly monotone:

(ii) A is V-Lipschitz mapping;

(iii) there exists a real number $\xi>0$ such that

$$
\xi \phi^{2}(t)-\frac{\nu^{2}(t)}{\xi}+\frac{2 \nu(t)}{\xi}<-2 \Psi(t)<\frac{1}{\xi}+\xi \phi^{2}(t)
$$

Then the M.N.C.P. (2.1) has a unique solution.

COROLIARY 3.2. Assume that

(i) $T$ is $\Psi$-strongly monotone mapping with respect to $G$;

(ii) $G$ is an expansive mapping, that is, there exists $\lambda \geq 1$ such that ||$G(x)-G(y)|| \geq \lambda|| x-y \|$. for all $\mathrm{x}, \mathrm{y} \in \mathrm{D}$ :

(iii) $\|T(x)-T(y)\| \leq \| x-y|| \phi(\|G(x)-G(y)\|)$, for all $x, y \in D$ :

(iv) $\|A(x)-A(y)\| \leq \| x-y|| v(\|G(x)-G(y)\|)$, for all $x, y \in D_{i}$

(v) there exists a real number $\xi>0$ such that, $\xi \phi^{2}(t)-\frac{\nu^{2}(t)}{\xi}+\frac{2 \nu(t)}{\xi}<2 \Psi(t)<\frac{1}{\xi}+\xi \phi^{2}(t)$

for all $t \in R_{+}$.

Then the S.N.I.C.P. has a unique solution.

REMARK 3.1. If $A=0$ then Theorem 3.1, Corollary 3.1 and Corollary 3.2 reduce to Theorem 2, Corollary 2 and Corollary 4, respectively, Isac [9].

4. ALGORITHM

In this section we give an iterative algorithm for finding the approximate solution of the S.N.I.C.P. and prove that the approximate solution converges to the exact solution. For this, we need the following result.

LEMMA 4.1. If $\mathrm{K}$ is a convex cone in $\mathrm{H}, \mathrm{x} \varepsilon \mathrm{H}$ is a solution of the S.N.I.C.P. if 
and only if it satisfies the relation

where

$$
\mathbf{x}=\mathbf{F}(\mathbf{x}),
$$

and $\xi>0$ is a constant.

PROOF. If follows directly from Proposition 2.1.

In view of this lemma, we suggest the following new unified algorithm for finding the approximate solution of the S.N.I.C.P.

ALGORITHU 4.1. For any given $x_{0} \varepsilon H$, compute $x_{n+1}$ by the iterative scheme

$$
x_{n+1}=x_{n}-G\left(x_{n}\right)+P_{K}\left[G\left(x_{n}\right)-\xi\left(T\left(x_{n}\right)+A\left(x_{n}\right)\right)\right] \text {. }
$$

$\mathrm{n}=0,1,2, \ldots$

where $\xi>0$ is a constant.

In order to discuss the convergence properties of the Algorithm 4.1 , we need the following concepts.

DEFINITION 4.1. An operator $\mathrm{T}: \mathrm{K} \rightarrow \mathrm{H}$ is called:

(i) Coercive if there exists a constant $\alpha>0$ such that

$$
\left\langle T(x), x>\geq \alpha|| x||^{2}\right. \text {, }
$$

for all $x \in \mathrm{K}$;

(ii) Continuous (bounded), if there is a constant $B>0$ such that

$$
\langle\mathrm{T}(\mathrm{x}), \mathrm{Y}\rangle \leq \beta|| \mathbf{x}\|\| \mathrm{y} \| \text {. }
$$

for all $x, y \in K$.

THEOREM 4.1. Let $T$ and $G$ be both Coercive and Continuous with coercivity constant $\alpha, \delta$ and continuity constants $\beta, \sigma$ respectively, and let $A$ be a Lipschitz continuous with constant $\mu$. If $x_{n+1}$ and $x$ are solutions of (4.2) and (2.3), respectively. then

$$
x_{n+1} \text { converges strongly to } x \text { in } H \text {, }
$$

for

$$
\begin{gathered}
\left|\xi-\frac{\alpha+\mu(k-1)}{\beta^{2}-\mu^{2}}\right|<\frac{\sqrt{(\alpha+\mu(k-1))^{2}-\left(\beta^{2}-\mu^{2}\right) k(2-k)}}{\beta^{2}-\mu^{2}} ; \\
\alpha>\mu(1-k)+\sqrt{\left(\beta^{2}-\mu^{2}\right) k(2-k) \cdot \beta^{2}-\mu^{2}>0 \text { and } \mu(1-k)<\alpha} \\
k=2 \sqrt{\left(1-2 \delta+\sigma^{2}\right)}<1
\end{gathered}
$$

PROOF. By Lemma 4.1, we see that the solution of the S.N.I.C.P. can be characterized by $(4.1)$. Hence from $(4.1)$ and $(4.2)$, we have

$$
\begin{aligned}
|| x_{n+1}-x|| & =|| x_{n}-G\left(x_{n}\right)+P_{K}\left[G\left(x_{n}\right)-\xi\left(T\left(x_{n}\right)+A\left(x_{n}\right)\right)\right]-x+G(x)-P_{K}[G(x)-\xi(T(x)+A(x))] \| \\
& \leq|| x_{n}-x-\left(G\left(x_{n}\right)-G(x)\right)||+|| G\left(x_{n}\right)-\xi\left(T\left(x_{n}\right)+A\left(x_{n}\right)\right)-G(x)+\xi(T(x)+A(x))|| .
\end{aligned}
$$

Since $P_{K}$ is nonexpansive.

$$
\left.\leq 2|| x_{n}-x-\left(G\left(x_{n}\right)-G(x)\right)||+|| x_{n}-x-\xi\left(T\left(x_{n}\right)-T(x)\right)||+\xi|| A\left(x_{n}\right)-A(x)\right)||
$$

By using the method of Noor [12] and, coercivity and continuity of $T$ and G, we get 


$$
|| x_{n}-x-\left(G\left(x_{n}\right)-G(x)\right)|| \leq \sqrt{\left(1-2 \delta+\sigma^{2}\right)}|| x_{n}-x||
$$

and

$$
\left\|x_{n}-x-\xi\left(T\left(x_{n}\right)-T(x)\right)\right\| \leq \sqrt{\left(1-2 \alpha \xi+B^{2} \xi^{2}\right)} \| x_{n}-x||
$$

Therefore, from (4.3), (4.4) and (4.5) and by using the Lipschitz continuity of A, we have

$$
\begin{aligned}
&\left\|x_{n+1}-x\right\| \leq\left[2 \sqrt{\left(1-2 \delta+\sigma^{2}\right)}+\sqrt{\left(1-2 \alpha \xi+\beta^{2} \xi^{2}\right)}+\mu \xi\right]\left\|x_{n}-x\right\| \\
&=\{\mu \xi+k+t(\xi)\} \\
& t(\xi)= \sqrt{\left(1-2 \alpha \xi+\beta^{2} \xi^{2}\right)} \\
&|| x_{n+1}-x \|=\text {, where } k=2 \sqrt{\left(1-2 \delta+\sigma^{2}\right)} \quad \text { is a constant and } \\
& t \mid x_{n-x} \| \text {, where } \theta=k+t(\xi)+\mu \xi
\end{aligned}
$$

Now we have to show that $\theta<1$. For this, we assume that the minimum value of $t(\xi)$ at $\bar{\xi}=\frac{\alpha}{\beta^{2}}$ with $t(\bar{\xi})=\sqrt{\left(1-\frac{\alpha^{2}}{\beta^{2}}\right.}$. For $\xi=\bar{\xi}, k+t(\bar{\xi})+\mu \xi<$ implies that $k<1$ and

$$
\alpha>\mu(1-k)+\sqrt{\left(B^{2}-\mu^{2}\right) k(2-k)}
$$

Thus, it follow that $\theta=k+t(\xi)+\mu \xi<l$ for all $\xi$ with

$$
\begin{aligned}
& \left|\xi-\frac{\alpha+\mu(k-1)}{\beta^{2}-\mu^{2}}\right|<\frac{\sqrt{(\alpha+\mu(k-1))^{2}-\left(\beta^{2}-\mu^{2}\right) k(2-k)}}{\beta^{2}-\mu^{2}} \\
& k<1, \beta^{2}-\mu^{2}>0, \\
& \alpha>\mu(1-k)+\sqrt{\left(\beta^{2}-\mu^{2}\right) k(2-k)} \text { and } \mu(1-k)<\alpha .
\end{aligned}
$$

Since $\theta<1$, the fixed point problem (4.1) has a unique solution $x$ and consequently the Picard iterates $x_{n+1}$ converges to $x$ strongly in $H$.

REMARK 4.1. (i) If the nonlinear operator $A$ is independent of $x$, that is, $A(x)=0$, then Algorithm 4.1 reduces to Algorithm 3.1 [16].

(ii) If $G$ is the identity operator, that is, $G(x)=x$, then Algorithm 4.1 reduces to Algorithm 2.1 [12].

\section{REFEREACES}

1. COTTLE, R.W. Nonlinear programs with positively bounded Jacobians, SIAM J. Appl. Math. 14 (1966), 147-157.

2. COTTLE, R N. DANTZIG, G.B. Complementary pivot theory of Mathematical programming, Linear Algebra and Appl. 1 (1968), 103-125.

3. BENSOUSSAN, A., COURSET, M. \& LIONS, J.L. Contrôle impulsionnel et inequation quasi-variationnelles stationnaries, C.R. Acad. Sci. Ser. I, Math. Paris 276 (1973), 1279-1284.

4. BENSOUSSAN, A. \& LIONS, J.L. Mouvelle formulation des problèmes de contrôle impulsionnel et applications, C.R. Acad. Sci. Ser. I, Math. Paris 276 (1973), 1189-1192.

5. BENSOUSSAN, A. \& LIONS, J.L. Problèmes de temps d'arrêt optimal et inequations variationnelles paraboliques, Applicable Anal. (1973), 267-194. 
6. BENSOUSSAN, A. LIONS, J.L. Nouvelles méthodes en contrôle impulsionnel, Anpl. Math. Optim. 1 (1975), 289-312.

7. DOLCETTA, I.C. MOSCO, U. Implicit complementarity problems and quasi-variational inequalities, In: R.W. Cottle, F. Giannessi and J.L. Lions eds: "Variational Inequalities and Complementarity Problems, Theory and Applications" pp. 75-87, John Wiley and Sons, New York, 1980.

8. ISAC, G. On the implicit complementarity problem in Hilbert spaces, Bull. Austral. Math. Soc., 32 (1985), 251-260.

9. ISAC, G. Fixed point theory and complementarity problem in Hilbert spaces, Bull. Austral. Math. Soc., 36 (1987), 295-310.

10. ISAC, G. Fixed point theory, coincidence equations on convex cones and complementarity problem, Contemporary Math. 72 (1988), 139-155.

11. Mosco, $\dot{U}$. On some nonlinear quasivariational inequalities and implicit complementarity problems in stochastic control theory, In: R.W. Cottle, F. Giannessi and J.L. Lions eds: "Variational Inequalities and complementarity problems, Theory and Applications", pp. 271-283, John Wiley and Sons, New York, 1980.

12. NOOR, M.A. On the nonlinear complementarity problem, J. Math. Anal. Appl. 123 (1987), 455-460.

13. ZARANTONELLO, E.H. Projections on convex sets in Hilbert space and spectral theory, In: E.H. Zarantonello ed.: "Contribution to Nonlinear Functional Analysis" pp. 237-424, Academic Press, New York, 1971.

14. BOYD, D.W. \& WONG, J.S.W. On nonlinear contractions, Proc. Amer. Math. Soc.. 20 (1969), 458-464.

15. KINDERLEHRAR, D. \& STAMPACCHIA, G. "An Introduction to Variational Inequalities and their Applications". Academic Press, New York, 1980.

16. NOOR, M.A. On complementarity problems, Math. Japonica 34 (1989), 83-88.

17. NOOR, M.A. General variational Inequalities, Appl. Math. Lett. 1 (1988).

18. NOOR, M.A. Quasi variational inequalities, Appl. Math. Lett. 1 (1988), 367-370. 


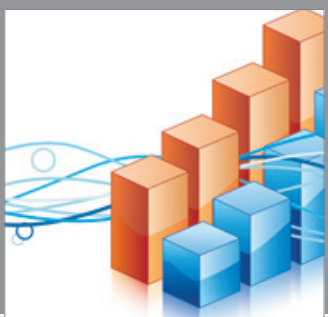

Advances in

Operations Research

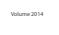

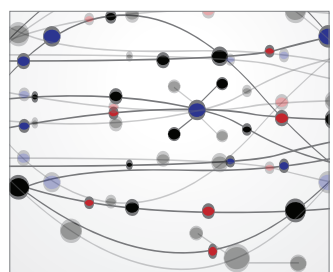

\section{The Scientific} World Journal
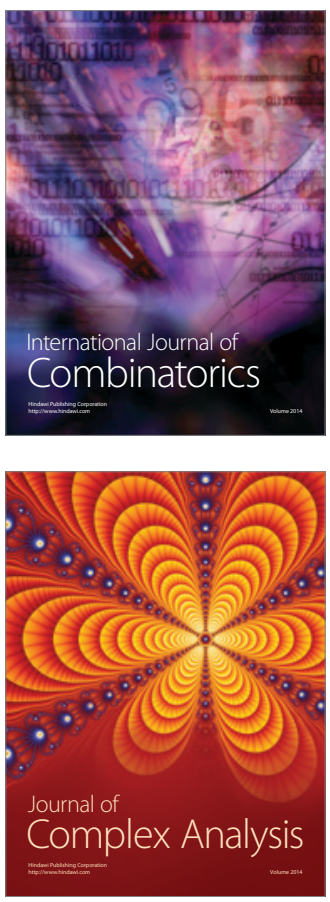

International Journal of

Mathematics and

Mathematical

Sciences
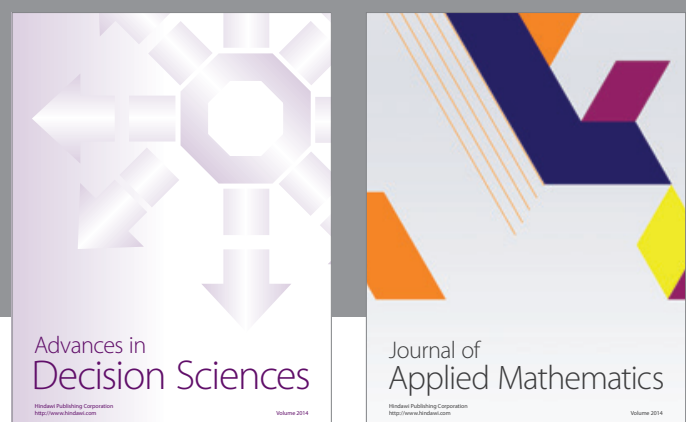

Journal of

Applied Mathematics
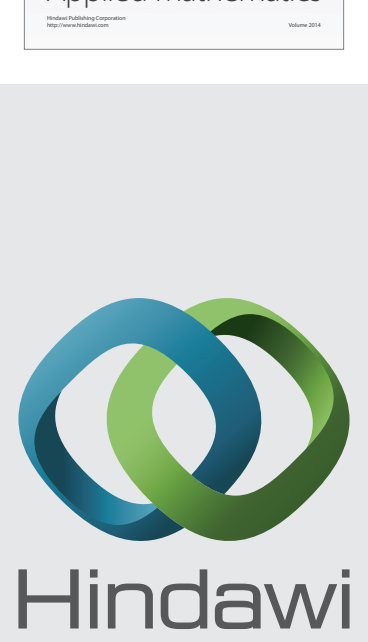

Submit your manuscripts at http://www.hindawi.com
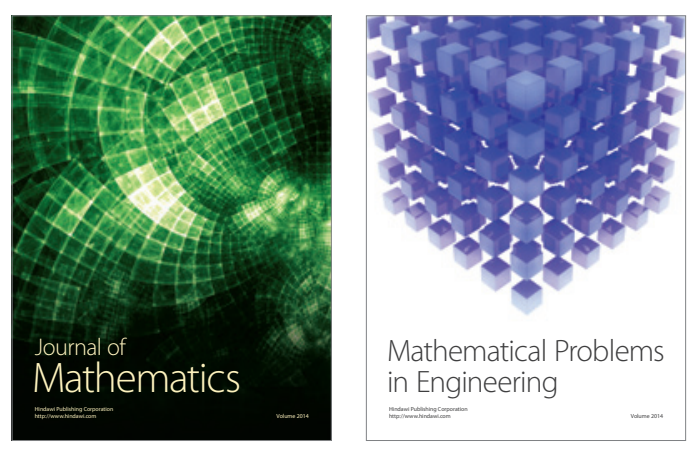

Mathematical Problems in Engineering
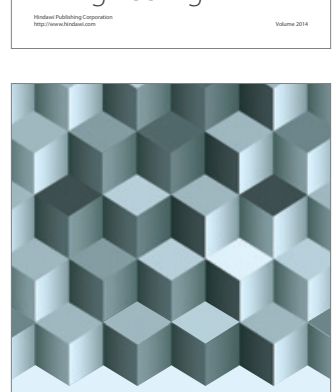

Journal of

Function Spaces
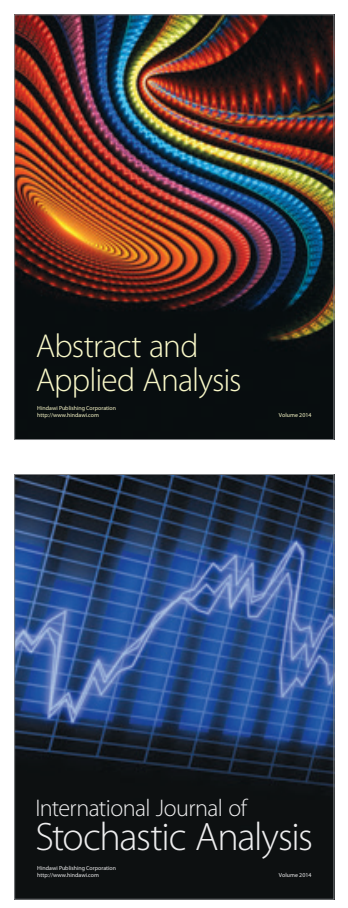

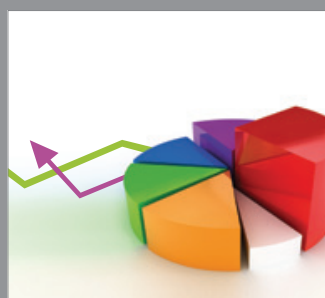

ournal of

Probability and Statistics

Promensencen
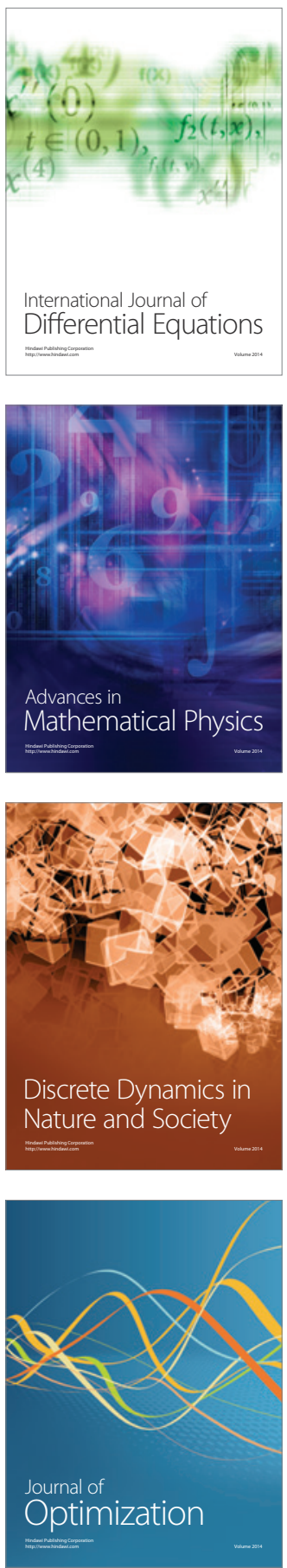